\title{
Effects of Broken Skirts and Pollution on Voltage Distribution for Cap and Pin Glass Insulators
}

\author{
C. Mateus \\ Instituto Politécnico de Lisboa (IPL), \\ Instituto Superior de Engenharia de \\ Lisboa (ISEL), Electrical, Energy and \\ Automation Department (ADEEEA) \\ Lisbon, Portugal \\ A44397@alunos.isel.pt
}

\author{
F. A. Barata \\ Instituto Politécnico de Lisboa (IPL), \\ Instituto Superior de Engenharia de \\ Lisboa (ISEL), Electrical, Energy and \\ Automation Department (ADEEEA) \\ Lisbon, Portugal \\ fbarata@deea.isel.ipl.pt
}

\author{
R. Luís \\ Instituto Politécnico de Lisboa (IPL), \\ Instituto Superior de Engenharia de \\ Lisboa (ISEL), Electrical, Energy and \\ Automation Department (ADEEEA) \\ Lisbon, Portugal \\ ricardo.luis@isel.pt
}

\begin{abstract}
Cap and Pin insulators are key elements on aerial electric power transmission lines in which their performance is subject to the environmental elements, like pollution and/or humidity. Furthermore, a broken insulator (stub) on an insulator string may change the voltage distribution along the chain. This paper goal is to study the effects of the voltage distribution along with tempered glass cap and pin insulator string in the presence of broken skirts as well contamination by pollution, using for that purpose a finite element software. This paper presents the voltage distribution profile across single and fourteen units of glass insulator string with stubs at distinct locations as well for the presence of contamination by pollution. It is concluded that the presence of pollution on a string with stubs will alter and exacerbate the increase of electrical potential effect due to the presence of stubs.
\end{abstract}

Keywords - Glass insulator string; stub; voltage distribution; pollution, finite element software.

\section{INTRODUCTION}

Due to the better knowledge regarding the materials characteristic's parameterization used in broken and polluted insulators, simulated studies had become similar to studies with an experimental component. Recent studies with numerical simulation models of insulators demonstrate the validity of numerical techniques to estimate the electrical field and potential distribution on insulators such as the Boundary Element Method (BEM), the Finite Difference Method (FDM) and the, Finite Element Method (FEM), [1, 2]. Generally, in engineering, the FEM has already been established for a long time and is a successful numerical method to solve electric field problems, using the discretization of the domain, as well as coupling with other physical processes (multi-physics), [2, 3].

Both experimental and simulated studies, concluded that location and stubs number will affect the string electrical breakdown. Some authors analysed that the worst-case scenario is when stubs are consecutively positioned closer to the beginning of the string, $[4,5]$. On the other hand, the voltage distribution is also affected by the supporting structure due to the capacitance between the support and the insulator, [6].

In contamination with pollution context, experimental and simulated studies, with a good degree of certainty have been developed to understand the voltage distribution on insulator strings, [7-9]. The study [7] was conducted in three scenarios: "fully, bottom and upper pollution". For each scenario, they included simulated and experimental measurements. In [10], authors also studied the pollution factor, comparing with simulations between them a string of seven insulators in three distinct ways "no-pollution, light, moderate and heavy pollution. An experimental studied was conducted in [11], with tests made on stubs individually or in full strings under several environmental elements (dry, wet or humid conditions).

Although important conclusions have been obtained in the two separate contexts (stubs and pollution), the two anomalies conjugation have not yet been analyzed, being this study objective these two factors combination .

As such, taking into account the materials electrical characteristics that constitute an insulator, a complete string (14 units - typical string for $220 \mathrm{kV}$ insulation level on AC power transmission lines) voltage distribution profile is presented, simulating the presence of two stubs in $1^{\text {st }}$ and $2^{\text {nd }}$ position (GND end) with a partial pollution (only in the lower side of the insulators) and with a full polluted string. The partial pollution simulation goal is to simulate the pollution effects in a string that was cleaned after rain, keeping the lower side pollution layer.

The next sections of this paper are organized as follows: In Section II the 2D finite element model for a glass insulator is presented, where the materials properties, boundary conditions and the finite element mesh are depicted. Section III presents the simulation results and discussion for one insulator and to an insulator string without/with stubs, and also with three distinguished situations, clean, partial pollution and total pollution. Finally, the main conclusions are summarized in Section IV.

\section{FINITE ELEMENT MODEL}

Cap and pin HV glass suspension insulators U160BS type (mostly used in Portugal for $220 \mathrm{kV}$ and $400 \mathrm{kV}$ transmission lines) is modelled in this study, Fig.1, the technical parameters can be seen in more detail in [12] and summarized in Table I and Table II. 
TABLE I.

\begin{tabular}{|l|c|c|}
\hline Insulator/Stub & U160BS & Stub \\
\hline Nominal creepage distance [mm] & 380 & 34.77 \\
\hline Shed diameter, D [mm] & 280 & N/A \\
\hline Suspension length, P [mm] & 146 & 146 \\
\hline Step, d [mm] & 20 & 20 \\
\hline
\end{tabular}

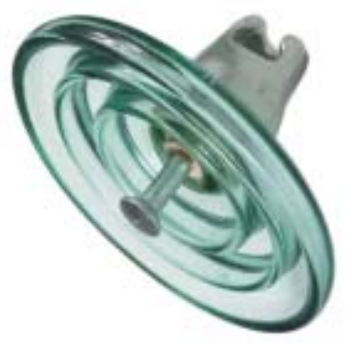

(a)

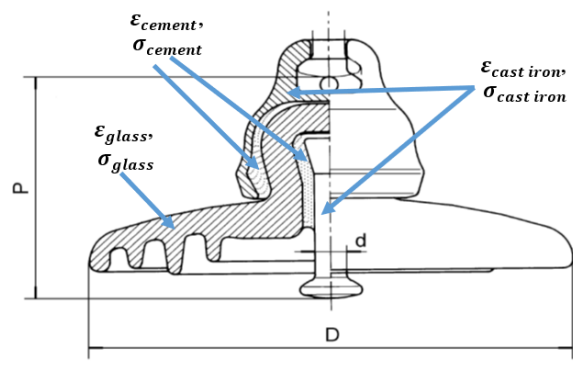

(b)

Fig. 1. Glass insulator U160BS: (a) photo of cap and pin glass insulator; (b) Types of materials in the insulator.

The simulation analysis was conducted using FEMMC finite element analysis (FEA) software, [13], firstly to comprehend the glass insulator behaviour for 1-unit, and then for 14-units. The geometric model of each glass string is represented in the twodimensional axisymmetric domain and the simulation is performed in steady-state current flow analysis (AC conduction study) using the material properties presented in Table II.

TABLE II. MATERIAL PROPERTIES

\begin{tabular}{|c|c|c|}
\hline $\begin{array}{c}\text { Type of } \\
\text { material }\end{array}$ & $\begin{array}{c}\text { Relative permittivity, } \\
\boldsymbol{\varepsilon}_{\mathbf{r}}(\mathbf{F} / \mathbf{m})\end{array}$ & $\begin{array}{c}\text { Conductivity, } \\
\boldsymbol{\sigma}(\mathbf{S} / \mathbf{m})\end{array}$ \\
\hline Air & 1 & 0 \\
\hline Cement & 15 & 0.0001 \\
\hline Glass & 4.2 & 0 \\
\hline Cast Iron & 1000 & 104400 \\
\hline Pollution Layer & 81 & 0.01 \\
\hline
\end{tabular}

Fig. 2 presents the boundary conditions applied to the insulator, with $U=0 \mathrm{~V}$ in the insulator cap and $U=U_{s}$ in insulator pin, where the value of $U_{s}$ is determined by the number of the glass insulators string considered. The exterior boundary considers the Neumann boundary condition for the edges.

The finite element formulation to obtain the simulation results is based on $\mathrm{AC}$ conduction analysis in the electric field domain, by applying $\mathrm{AC}$ voltage to the insulator(s).

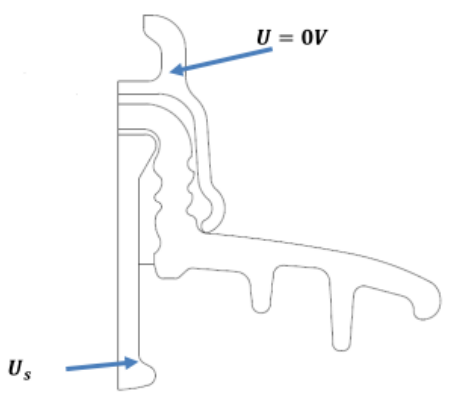

Fig. 2. Boundary conditions applied to the insulator.

The formulation is derived from Maxwell's equations, combining the equations for electrostatics (1) and AC conduction (2) and taking account the Ohm's law (3).

$$
\begin{gathered}
\nabla . \varepsilon \vec{E}=\rho \\
\nabla . \vec{J}=-j \omega \sigma \\
\vec{J}=\sigma \vec{E}
\end{gathered}
$$

Where:

$\sigma$ is electric conductivity of the material $(\mathrm{S} / \mathrm{m})$;

$\rho$ is electric charge density $\left(\mathrm{C} / \mathrm{m}^{3}\right)$;

$\varepsilon$ is dielectric constant of material;

$J$ is current density $\left(\mathrm{A} / \mathrm{m}^{2}\right)$;

$E$ is electric field strength $(\mathrm{V} / \mathrm{m})$;

$\omega$ is angular electric frequency $(\mathrm{rad} / \mathrm{s})$.

The final equation with the respect to electric potential, $U$, to obtain the simulation results, under AC conduction analysis with $50 \mathrm{~Hz}$ frequency is (4).

$$
\nabla \cdot\left(\left[\varepsilon-\frac{j \sigma}{\omega}\right] \nabla U\right)=0
$$

Fig. 3 depicts the obtained finite element mesh of the insulator with 15973 nodes and 31527 elements, where each node will be processed with the finite element formulation present at (4).

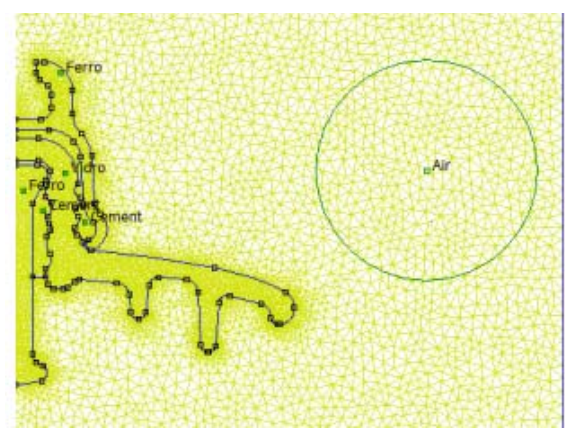

Fig. 3. Finite element mesh of the insulator. 


\section{FEA RESULTS AND DISCUSSION}

As mentioned, the simulation analysis was conducted using FEMMC firstly for 1-unit, and then for 14-units of glass insulators string.

\section{A. One insulator U160BS}

In this Section, it will be analyzed one isolated insulator behaviour in distinct situations. The insulator performance under the typical applied $11 \mathrm{kV}$ voltage [5] is compared with its stub and also with the insulator with/without pollution layer.

\section{1) Insulator $V_{S}$ Stub}

To understand the $s t u b$ effect in an insulator string, firstly the behaviour of one perfect isolated insulator, Fig. 4 and it stub, Fig. 5, is compared and the differences between them are characterized and analyzed.

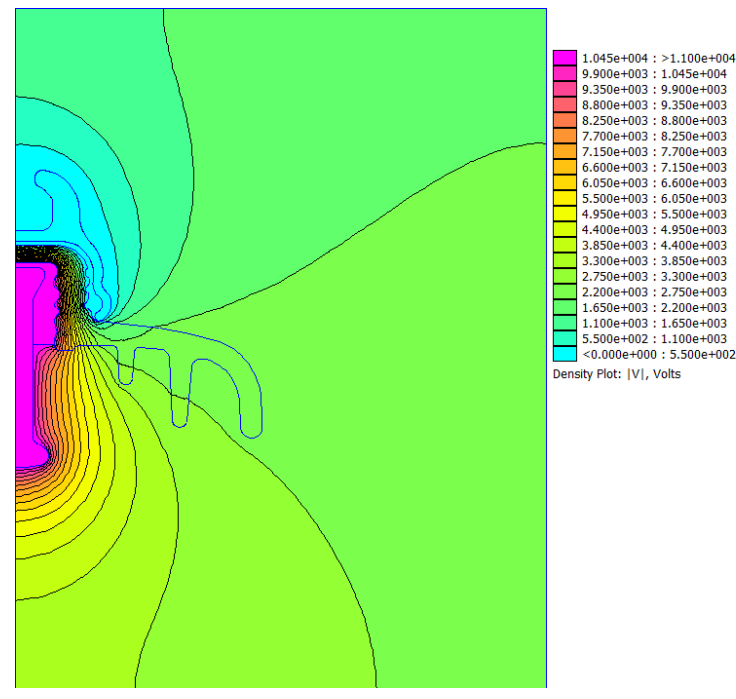

Fig. 4. Contour Profile for U160BS

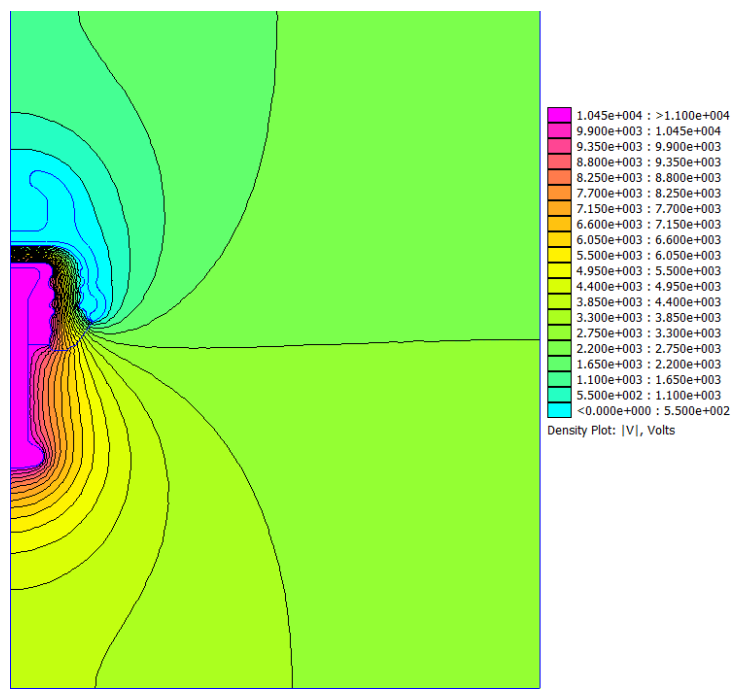

Fig. 5. Contour Profile for U160BS Stub.

Fig. 6, presents one insulator U160BS and stub voltage distribution profile, related with the FEA depicted in Fig. 4 and Fig. 5.

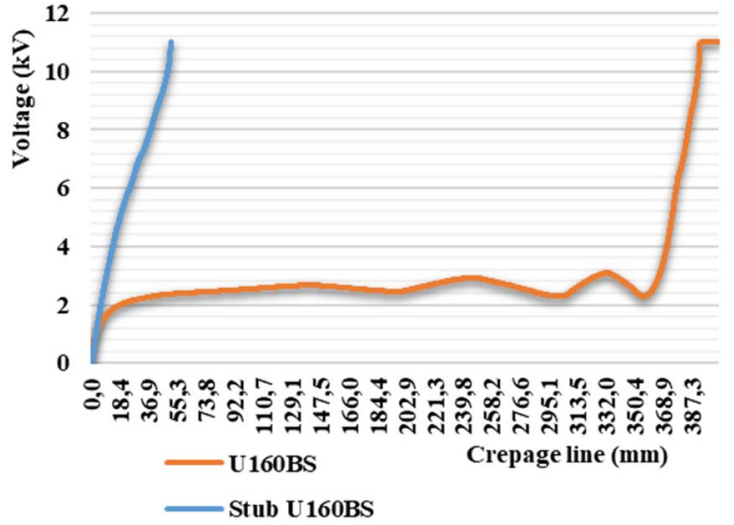

Fig. 6. Voltage Distribution Profile for U160BS vs Stub.

Analyzing Fig. 4-6, the most noticeable difference is undoubtedly the voltage evolution, due to the leakage line being much smaller in the stub. It should be noted that the final slope to the voltage applied to the pin is identical in the $s t u b$ and insulator, verifying the performance (or lack) of the dielectric ribs. It is also verified that the relationship between variables in the $s t u b$ it is developed in a more markedly when compared with the perfect insulator that is almost constant. It should be noted that, due to the insulator lower ribs lack, the voltage distribution along the $s t u b$ model is more linear, contrasting with the voltage distribution in a perfect insulator.

\section{2) Insulator with pollution layer}

In this section one isolated insulator U160BS under several conditions is simulated, clean, partial pollution and total pollution, Fig. 7 and Fig. 8. The pollution layer has $2 \mathrm{~mm}$ thickness, in which the "total pollution" refers for a uniform layer covering the entire contour of the dielectric and "partial pollution" covers only the lower contour.

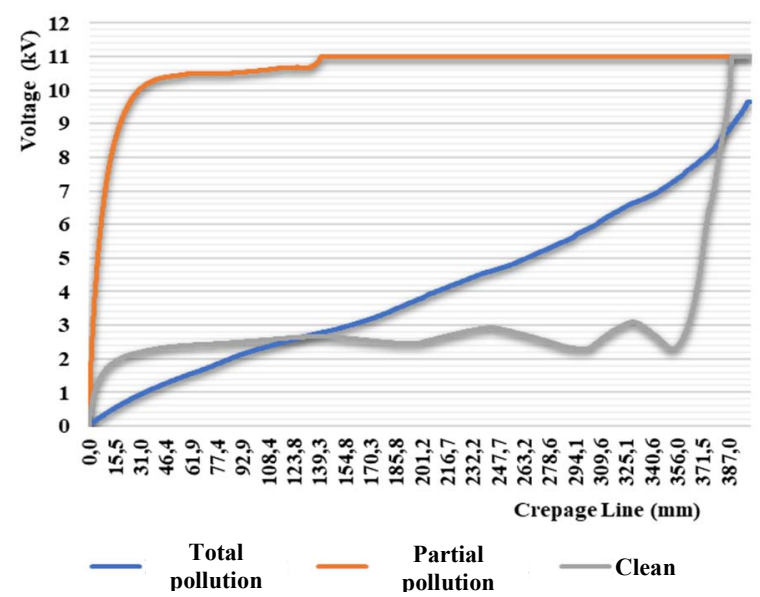

Fig. 7. Voltage Distribution Profile for for U160BS.

Comparing the three voltage distributions along the leakage line (clean, partial pollution and total pollution), Fig. 7, remarkable differences are observed regarding voltage evolution. The characteristic insulator voltage profile is transformed into a mostly resistive component with total pollution, and mostly capacitive with partial pollution. 


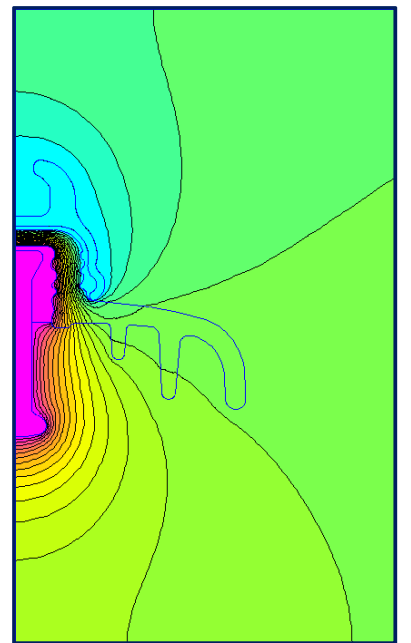

(a)

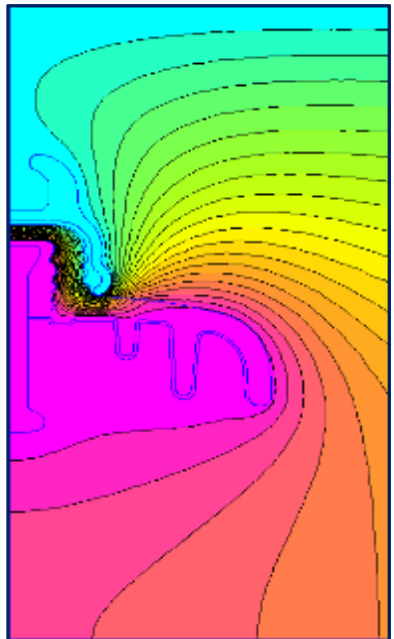

(b)

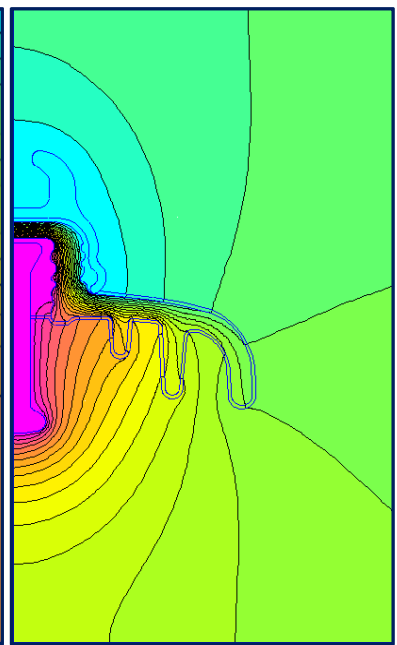

$1.045 e+004:>1.100 e+004$ $9.900 \mathrm{e}+003: 1.045 \mathrm{e}+004$ $9.350 \mathrm{e}+003: 9.900 \mathrm{e}+003$ $8.800 \mathrm{e}+003: 9.350 \mathrm{e}+003$ $8.250 \mathrm{e}+003: 8.800 \mathrm{e}+003$ $7.700 \mathrm{e}+003: 8.250 \mathrm{e}+003$ $7.150 \mathrm{e}+003: 7.700 \mathrm{e}+003$ $6.600 \mathrm{e}+003: 7.150 \mathrm{e}+003$ $6.050 \mathrm{e}+003: 6.600 \mathrm{e}+003$ $5.500 e+003: 6.050 e+003$ $4.4000+003: 4.950 e+003$ $4.400 \mathrm{e}+003: 4.950 \mathrm{e}+003$ $3.850 \mathrm{e}+003: 4.400 \mathrm{e}+003$ $3.300 \mathrm{e}+003: 3.850 \mathrm{e}+003$ $2.750 \mathrm{e}+003: 3.300 \mathrm{e}+003$ $2.200 e+003: 2.750 e+003$ .650e+003: $: 2.200 e+003$ $1.100 \mathrm{e}+003: 1.650 \mathrm{e}+003$ $5.500 \mathrm{e}+002: 1.100 \mathrm{e}+003$ $<0.000 \mathrm{e}+000: 5.500 \mathrm{e}+002$ Density Plot: $|\mathrm{V}|$, Volts

Fig. 8. Contour Profile for U160BS: (a) Clean insulator ; (b) Partial Pollution; (c) Total Pollution.

With the pollution layer involving the entire dielectric (Total Pollution), it is emphasized the presence of equipotential lines inside the dielectric, which did not exist in the case of the clean insulator, Fig. 8c. This effect is due to the pollution layer electrical conductivity and permittivity. The voltage distribution along the leakage line in the cement zone has the shape of a linear function, Fig. 7, and it can be inferred by this fact, that the voltage distribution has a predominantly resistive influence.

Considering the location of the leakage line and where the highest voltage $(11 \mathrm{kV})$ is located, it is determined that, both for the clean insulator and for the insulator with total pollution, this location is significantly at the same point, at about $300 \mathrm{~mm}$ (direction pin), however, the evolution of the tension of the total polluted insulator is linear.

In the insulator case with partial pollution, the maximum voltage is located at $135 \mathrm{~mm}$, concluding that, from the isolation point of view, this is the worst of the two situations studied due to the higher isolation effect inhibition due to the dielectric.

\section{B. String of fourteen insulators}

In this section, an adaptation of the FEA referred on [5], with an AC single-phase voltage of $127 \mathrm{kV}$ stress is applied to the pin at the bottom of string insulator and the top insulator cap is grounded.

Bearing in mind that the insulator string has a symmetrical form, the simulation work was performed in an axisymmetric 2D model class. In this class, the cross-section of the insulator shown in Fig. 1 was adequate to represent the $3 \mathrm{D}$ modelling model using this software. It is important noting that the $s t u b$ was modelled with the creepage distance of $35 \mathrm{~mm}$. In addition, the supporting structures, conductors and other accessories are to be neglected in this study.

1) String of fourteen insulators, with twelve insulators in perfect conditions and two stubs near the GND end.

Voltage distribution profile for a complete string and a string with two stubs on the $1^{\text {st }}$ and $2^{\text {nd }}$ position is illustrated in Fig. 9.
It is clear from the figure that the voltage is distributed progressively along the creepage line for complete string. Nevertheless, the voltage distribution profile comes to increases after the stubs, comparatively to a complete string voltage distribution .

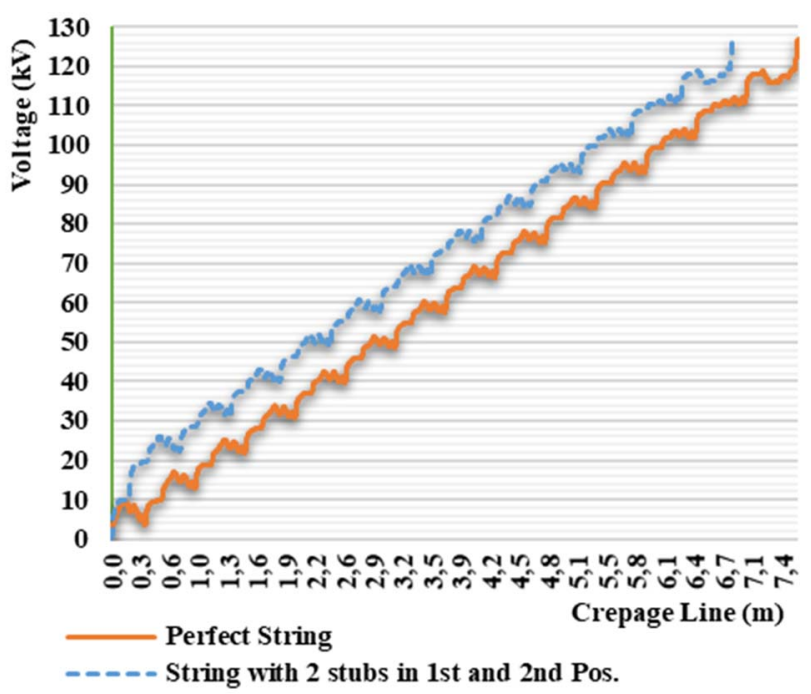

Fig. 9. Voltage Distribution Profile for Simulation A.

The percentage voltage increases in the insulator cap on the $3^{\text {rd }}$ Position is nearly $40 \%$, similarly to the results on [5].

\section{2) String with fourteen insulators with an added pollution} layer.

The pollution layer has $2 \mathrm{~mm}$ thickness, in which the "Total pollution" refers for a uniform layer covering the entire contour of the dielectric and "Partial Pollution" covers only the lower contour. The simulation parameters used for Section III.B.1) are the same for this section.

The contour profile of a Perfect String and for a string with Partial Pollution and Total Pollution are presented in Fig. 10. It 
is verified that the voltage level intensity, as well as equipotential lines density, increase gradually from Perfect String to Total Pollution.

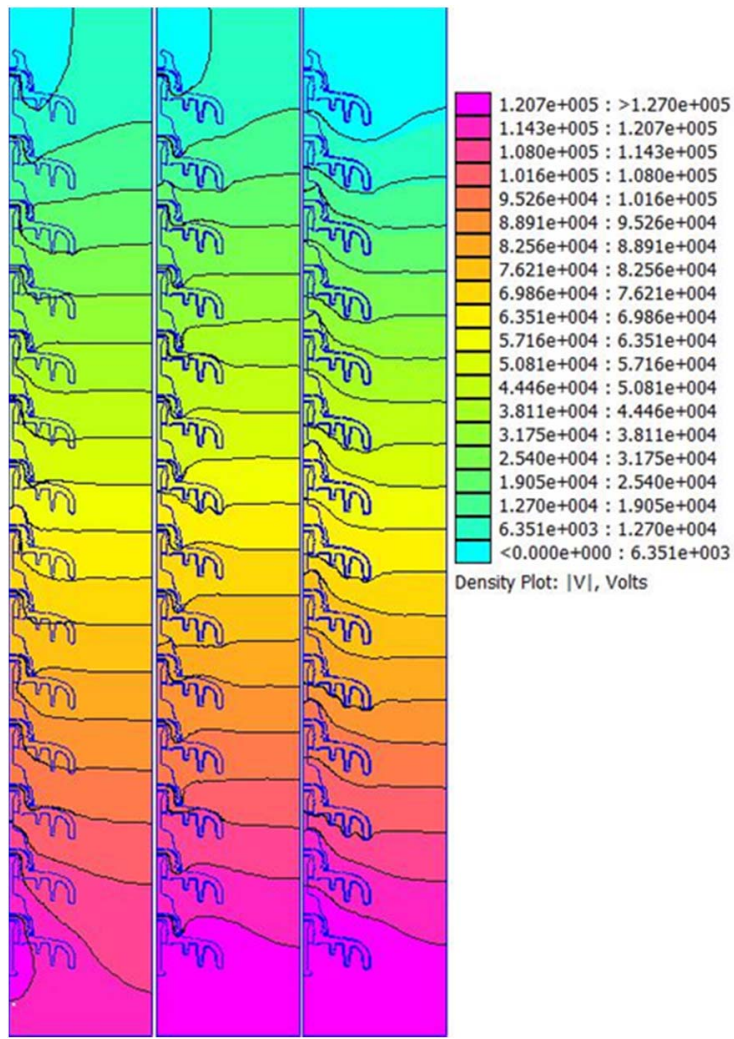

(a)

(b)

(c)

Fig. 10. Contour Profile for string of fourteen insulators, with twelve insulators in perfect conditions and two stubs near the GND end (a) Clean String (b) Partial Pollution (c) Total pollution.

Analysing Fig. 11 can be verified that the Voltage Distribution Profile, is affected by the pollution layer conductivity as well as its location.

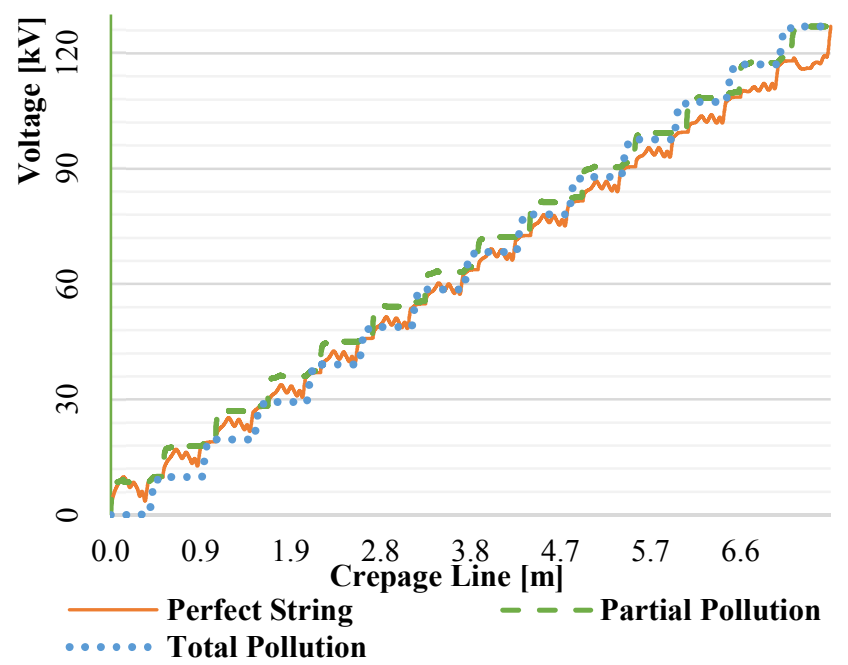

Fig. 11. Voltage Distribution Profile for a string with fourteen insulators with an added pollution layer.
According to [7], with Total Pollution, it is explained by the highly resistive field conduction, as result of the conductivity, the GND is extended to the entire dielectric of the 1 st insulator and the applied voltage $(127 \mathrm{kV})$ to the $14 \mathrm{t}^{\mathrm{h}}$ insulator. This consequence compresses the distribution to the remaining 12 insulators, inhibiting the insulation function of the $1^{\text {st }}$ and $14^{\text {th }}$.

For Partial Pollution, in which the bottom pollution layer is an equipotential surface, as Total Pollution, but the upper surface of the dielectric presents a voltage distribution like the perfect string, for the same area.

3) String of fourteen insulators, with an added pollution layer, and two stubs near the GND end.

In this section is simulated the parameters combination presented in Section III.B.1) and III.B.2), with a string with two stubs near the GND end in three situations, "Clean String", with "Partial Pollution" and with "Total Pollution". The voltage distribution profile for the three situations is represented in Fig. 12 and is worth mentioning that with Total Pollution is the worst-case scenario which it conjugates the presence of stubs in a total polluted string.

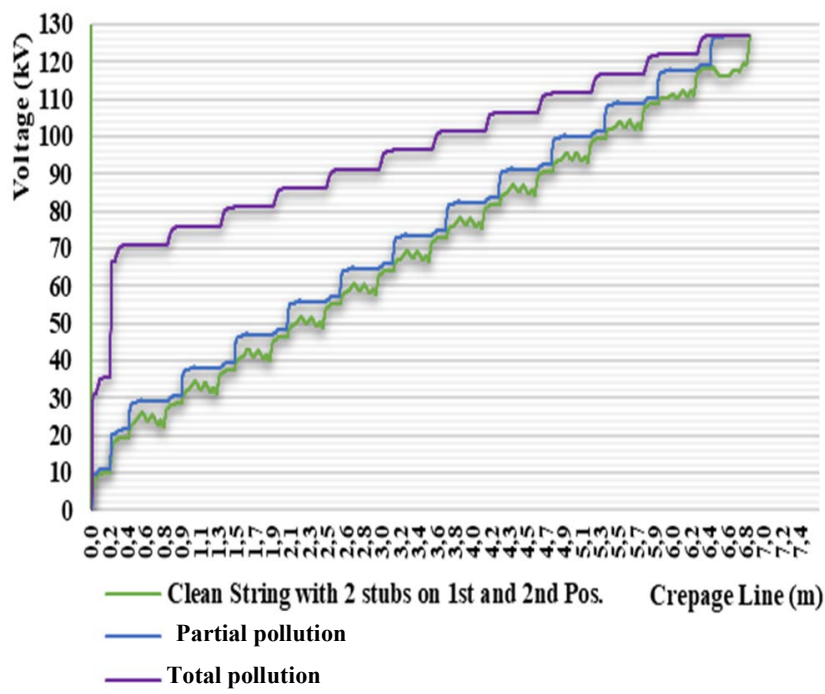

Fig. 12. Voltage Distribution Profile for a string of fourteen insulators, with an added pollution layer, and two stubs near the GND end.

It is verified that the voltage level intensity, as well as equipotential lines density, are slightly increased from Fig. 13a to Fig. 13b. In Fig. 13b, for Partial Pollution, it is clear an voltage distribution increase in comparison with Clean String with two stubs, with relevance to similar values in the lower contour as noticed in III.B.2). However, the most significant variation is between Partial Pollution and Total pollution. Due to the conductivity of the pollution layer in the entire surface of the dielectric, the top surface of the dielectric, it is noticed that the most part of the voltage drop is made on the stubs. This statement is confirmed by the Voltage Distribution profile, on Fig. 12, as is verified a voltage drop of approximately $70 \%$ of the applied voltage on the two stubs and $30 \%$ on the remaining perfect insulators. 


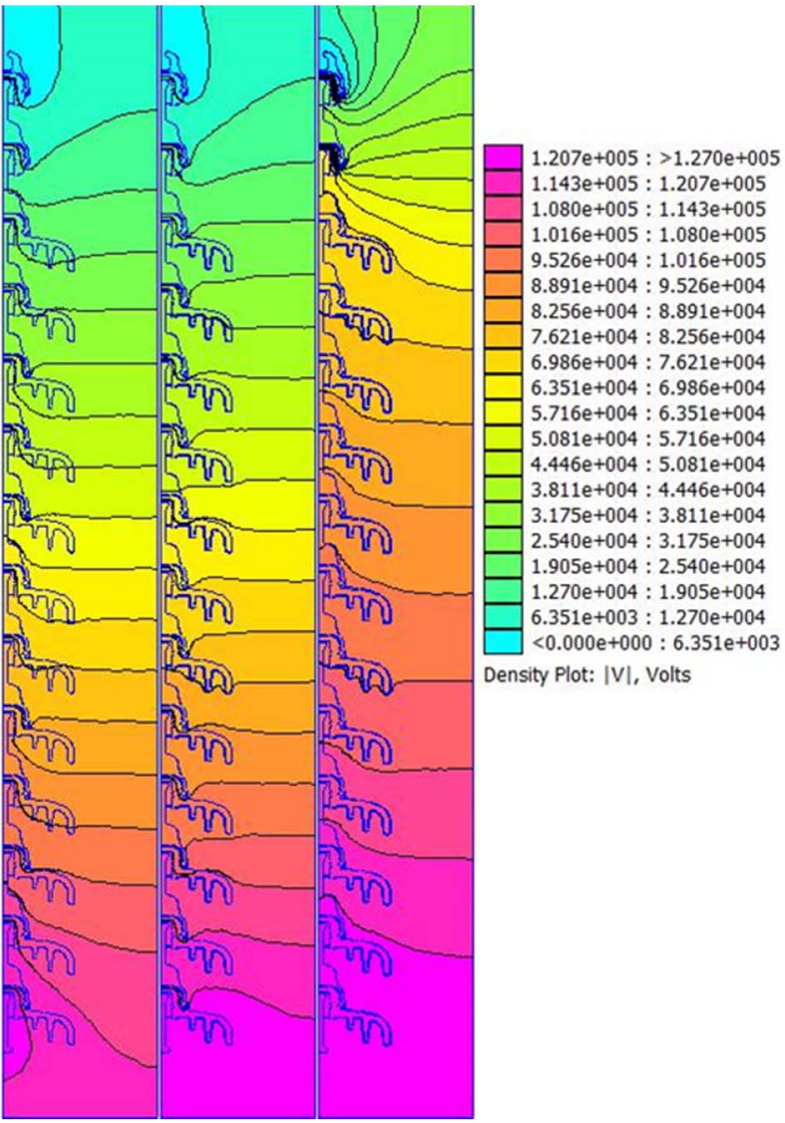

(a)

(b)

(c)

Fig. 13. Contour Profile for a string of fourteen insulators, with an added pollution layer, and two stubs near the GND end (a) Clean String (b) Partial Pollution (c) Total pollution.

\section{CONCLUSIONS}

To understand the role of one insulator in the entire string, the paper started by analysing firstly the behaviour of one isolated insulator under several conditions. The U160BS insulator was compared with its $s t u b$ and results showed that, due to the lack of the insulator lower ribs, the voltage distribution along the $s t u b$ model is more linear, contrasting with the voltage distribution in a perfect insulator. Comparing the voltage distributions along the leakage line with, a clean insulator and an insulator with total pollution and partial pollution, it can be concluded by the results analysis, that the insulator behaves in a resistive component with is total pollution and as a capacitive with partial pollution.

Then, voltage distribution profile across fourteen units of glass insulators string with stubs in clean and in polluted conditions was also performed. From results, it can be concluded that the pollution presence alters and exacerbates the stubs effect in the voltage distribution profile along the glass insulator string, being the worst-case for total polluted conditions.
Despite the achieved results, more work in this matter should be accomplished, namely, a comparison between insulators strings in transmission lines in Portugal for $150 \mathrm{kV}, 220 \mathrm{kV}$ and $400 \mathrm{kV}$, and a comparison between different dielectric materials characteristics should be archived. It is also suggested to simulate dry, wet and ice insulators. To obtain more accurate models, it is also important that more laboratory work is published on this topic, as well as the parameterization used in the materials characterization e.g., conductivity, permittivity, type of pollution and pollution layers dimension.

\section{REFERENCES}

[1] C. Muniraj, Chandrasekar Dr.S., "Finite element modeling for electric field and voltage distribution along the polluted polymeric insulator", World Jornal of Modelling and Simulation, vol. 8, no. 4, pp. 310-320, Jan. 2012.

[2] Benguesmia, Hani, Nassima M'ziou, and Ahmed Boubakeur, "Simulation of the potential and electric field distribution on high voltage insulator using the finite element method", Diagnostyka, vol. 19, no. 2, pp. 41-52, June 2018.

[3] Andreas Küchler, "High Voltage Engineering: Fundamentals Technology - Applications", Berlin, Germany, Springer Vieweg, 2018.

[4] L. Leite, J. A. Yanaguizawa, A. H. Shinohara, E. G. Costa, G. Xavier, and D. A. Maciel, "Experimental Study of Electrical Breakdown Voltage of a Glass Insulator Strings with Different Numbers of Broken Units", in Proceedings of the 2008 IEEE International Power Modulators and High Voltage Conference, Las Vegas, NV, USA, May 27-31, 2008, pp. 291294.

[5] N.A. Othman, M.A.M. Piah, Z. Adzis, "Effect of Broken Skirts on Voltage Distribution along Insulator Strings", International journal of simulation: systems, science \& technology, Vol. 17, no. 41, 2016, pp. 16.1-16.4.

[6] S. Ilhan, A. Ozdemir, S. H. Jayaram, and E. A. Cherney, "Ac and Transient Electric Field Distributions along a 380 kV V-String Insulator", IEEE International Symposium on Electrical Insulation, San Juan, PR, USA, June 10-13, 2012, pp. 3099-403.

[7] S. Ilhan, A. Ozdemir, S. H. Jayaram, and E. A. Cherney, "Simulations of Pollution and their Effects on the Electrical Performance of Glass Suspension Insulators," in 2012 Annual Report Conference on Electrical Insulation and Dielectric Phenomena (CEIDP), Montreal, QC, Canada , Oct. 14-17, 2012, pp. 803-806.

[8] N.A. Othman, M.A.M. Piah, Z. Adzis, H. Ahmad, N.A. Ahmad, "Simulation of Voltage and Electric-Field Distribution for Contaminated Glass Insulator", in 2013 IEEE Student Conference on Research and Development (SCOReD), Putrajaya, Malaysia, Dec. 16-17, 2013, pp.116120 .

[9] V.T. Kontargyri, I.F. Gonos, I.A. Stathopulos, A.M. Michaelides, "Calculation of the Electric Field on an Insulator string using the Finite Element Method", Proc. of the Universities Power Engineering Conference, Thessaloniki, Greece, Sep. 1-3, 2003, vol. 38, pp. 450-453.

[10] P. Unahalekhaka, S. Phonkaphon, "Simulation of Potential and Electric Field Due to Defective Insulator in $115 \mathrm{kV}$ Transmission Line", in GMSARN International Journal, Vol. 8, no. 1, pp. 1-6, Mar. 2014.

[11] J. M. George, S. Prat, S. Tartier, and Z. Lodi, "Electrical characteristics and properties of a stub (damaged toughened glass insulator)", $18^{\text {th }}$ International Symposium on High Voltage Engineering (ISH2013), Seoul, Korea, Aug. 25-30, 2013, pp.1155-1159.

[12] Sediver, "Toughened glass suspension insulators: IEC / BS / ANSI", technical catalogue, 2007.

[13] D. C. Meeker, FEMM - Finite Element Method Magnetics, Version 4.2, (28Feb2018 Build), http://www.femm.info. 